\title{
SCHAUDER BASES AND KÖTHE SEQUENCE SPACES
}

\author{
BY \\ ED DUBINSKY $\left({ }^{1}\right)$ AND J. R. RETHERFORD
}

In the light of the vast amount of literature on Schauder bases in Banach spaces which has appeared during the last thirty years, and considering the more recent development of the general theory of locally convex spaces, it is not surprising that many people are beginning to study Schauder bases in locally convex spaces. (See for example [1], [5], [7], [14], [16]-[21], [24].)

One of the most important areas in the field of locally convex spaces is the theory of Köthe sequence spaces [10]. Not very much is gained, of course, by looking at the sequence space determined by a locally convex space with a Schauder basis. However, if one considers the case in which the topological dual and the Köthe dual coincide, then the two theories seem to come together very nicely. One obtains easy proofs of old theorems and important generalizations of others. Of course, as might be expected in any study involving Köthe sequence spaces, one obtains many counterexamples which mark out the boundaries of possible theorems.

The authors feel that in this paper a new approach is introduced to both of the fields mentioned in the title and that although many new results are obtained, only the surface has been scratched. For example, no attempt has been made to study the specific types of locally convex spaces presently of interest to analysts, such as Fréchet spaces, $\mathscr{D} \mathscr{F}$-spaces, Montel spaces, and nuclear spaces. It should be mentioned, however, that in almost all cases, the hypotheses of our general theorems are satisfied by these spaces.

Aside from the overall approach, our main interest here is to study various types of Schauder bases which have previously been introduced, their relationships to each other and to various topological vector space concepts. In part I, we define the types of bases, interpret them in the context of a sequence space with a topology of uniform convergence on a family of subsets of the Köthe dual and determine when a locally convex space can be viewed in this context. In part II we study the types of bases and their implications. The most important result is Theorem (1.10) which is a generalization of a theorem of R. C. James [9]. Finally in part III, we apply our results to the concepts of reflexivity and similar bases.

Notation and terminology. If $E[\mathscr{T}]$; where $E$ is a vector space over the field $K$ of real or complex numbers and $\mathscr{T}$ is a topology on $E$; is a locally convex space (always assumed to be Hausdorff) and $\left(b^{n}\right)$ is a sequence in $E$, we say that $\left(b^{n}\right)$ is a

Received by the editors May 18, 1966.

(1) Research of the first author was supported, in part, by NSF Grant GP-3989. 
Schauder basis for $E[\mathscr{T}]$ if for each $x \in E$ there exists a unique sequence, $\left(x_{n}\right)$, in $K$ such that $x=\lim _{N} \sum_{n=1}^{N} x_{n} b^{n}$, and each of the maps, $f^{n}: E \rightarrow K$, defined by $f^{n}(x)=x_{n}$, are in $E^{\prime}$. When we mention a Schauder basis, $b^{n}$, we shall feel free to refer to $f^{n}$ as above without specific reference to the definition. In the few cases in which a Schauder basis is called something other than $\left(b^{n}\right)$, we shall explicitly mention the name of the corresponding $\left(f^{n}\right)$. Sometimes, $\left(f^{n}\right)$ will be referred to as the dual basis.

If $E, F$ are vector spaces over $K$, we shall say that $\langle E, F\rangle$ is a dual system if $\langle$,$\rangle is a bilinear function from E \times F$ to $K$ which "separates points" (see [4, p. 48]). If $x \in E$, then, we may consider $x$ as a linear functional on $F$ defined by $x(u)=\langle x, u\rangle$ and we shall interchange these two notations with abandon. Similarly for each $u \in F$. A dual system topology on, say, $E$ is a topology of uniform convergence on some class of subsets of $F$. Such a topology, $\mathscr{T}$, will be called compatible with the dual system if the topological dual $(E[\mathscr{T}])^{\prime}$ is $F$.

If $\Sigma$ is a family of subsets of $F$, we shall, following Köthe [10] denote the topology by $\mathscr{T}_{\Sigma}(F, E)$ and the locally convex space, by $E\left[\mathscr{T}_{\Sigma}(F, E)\right]$. When there is no possibility of confusion we shall shorten the notation to $\mathscr{T}_{\Sigma}(F), E\left[\mathscr{T}_{\Sigma}(F)\right]$ respectively. In this paper, we only use three cases which are the weak, Mackey, and strong topologies, denoted $\mathscr{T}_{s}(F, E), \mathscr{T}_{k}(F, E), \mathscr{T}_{b}(F, E)$ and defined by taking, $s=$ class of finite subsets of $F$

$k=$ class of all absolutely convex, $\mathscr{T}_{s}(E, F)$-compact subsets of $F$,

$b=$ class of all $\mathscr{T}_{s}(E, F)$-bounded subsets of $F$,

respectively. For such terms as absolutely convex, bounded, barrelled, equicontinuous, etc., we use the standard definitions as given, say, by Köthe [10].

If $V$ is a neighborhood of 0 in a locally convex space, $E[\mathscr{T}]$, we define the gage of $V$ to be the function, $\rho_{V}: E \rightarrow R$ defined by, $\rho_{V}(x)=\inf \left\{\lambda>0 \mid \lambda^{-1} x \notin V\right\}$.

A vector space, $\lambda$, of sequences of elements of $K$ will be called a sequence space if it contains all finitely nonzero sequences. If $\lambda$ is a sequence space, we define, following Köthe, two other sequence spaces, as follows,

$$
\begin{aligned}
\lambda^{\beta} & =\left\{u=\left(u_{i}\right) \mid \sum_{i=1}^{\infty} x_{i} u_{i} \text { converges for all } x=\left(x_{i}\right) \in \lambda\right\}, \\
\lambda^{\times} & =\left\{u=\left(u_{i}\right)\left|\sum_{i=1}^{\infty}\right| x_{i} u_{i} \mid \text { converges for all } x=\left(x_{i}\right) \in \lambda\right\} .
\end{aligned}
$$

It is obvious that $\lambda^{\beta}, \lambda^{\times}$are sequence spaces. The space $\lambda^{\times}$will be called the Köthe dual of $\lambda$. It is easy to see that both $\left\langle\lambda, \lambda^{\times}\right\rangle$and $\left\langle\lambda, \lambda^{\beta}\right\rangle$ are dual systems where $\langle x, u\rangle=\sum_{i=1}^{\infty} x_{i} u_{i}$ for $x \in \lambda$ and $u \in \lambda^{\beta}$ or $u \in \lambda^{\times}$.

Following Köthe we say that a sequence space, $\lambda$, is normal if $x \in \lambda$ and $\left|y_{i}\right| \leqq\left|x_{i}\right|$ implies $y=\left(y_{i}\right) \in \lambda$. We say $\lambda$ is perfect if $\lambda=\lambda^{\times x}$. Clearly every $\lambda^{\times}$is normal so perfect implies normal. If $x \in \lambda$, we define the $n$th (initial) section, $x^{n}$, of $x$ to be the sequence $\left(x_{i}^{n}\right)_{i}$ where $x_{i}^{n}=x_{i}$ for $i \leqq n$ and $x_{i}^{n}=0$ for $i>n$. If 
$N=\{1,2, \ldots\}$ and $J$ is a subsequence of $N$, we define the stepspace $\lambda_{J}$ of $\lambda$, corresponding to $J$ to be the sequence space

$\lambda_{J}=\left\{y=\left(y_{i}\right) \mid\right.$ there is an $x \in \lambda$ such that $y_{j}=x_{n}$, for all $\left.n_{j} \in J\right\}$.

The following specific examples of sequence spaces will be of interest to us:

$\varphi=\left\{x=\left(x_{i}\right) \mid x_{i}=0\right.$ for $i$ sufficiently large $\}$,

$\omega=\{$ all sequences in $K\}$,

$l^{1}=\left\{x=\left(x_{i}\right)\left|\sum_{i=1}^{\infty}\right| x_{i} \mid<\infty\right\}$,

$l^{\infty}=\left\{x=\left(x_{i}\right) \mid\right.$ there is an $M>0$ such that $\left|x_{i}\right| \leqq M$ for all $\left.i\right\}$,

$c_{0}=\left\{x=\left(x_{i}\right) \mid \lim _{i \rightarrow \infty} x_{i}=0\right\}$,

Clearly, $\varphi=\omega^{\times}, \omega=\varphi^{\times}, l^{1}=\left(l^{\infty}\right)^{\times}, l^{\infty}=\left(l^{1}\right)^{\times},\left(c_{0}\right)^{\times}=l^{1}$. We define $e^{n}$ to be the sequence such that $e_{i}^{n}=0$ if $i \neq n$ and $e_{n}^{n}=1$.

\section{TYPES OF BASES AND THEIR CORRESPONDING SEQUENCE SPACES}

\section{Types of bases.}

(1.1) First we define three types of bases which refer to the manner of convergence of the expansion of an element. We shall let $E=E[\mathscr{T}]$ be a locally convex space and $\left(b^{n}\right)$ a Schauder basis for $E$.

(i) we say that $\left(b^{n}\right)$ is unconditional if for each permutation $\pi$, on the natural numbers, $\sum_{n=1}^{\infty} f^{\pi(n)}(x) b^{\pi(n)} \in E$ for all $x \in E$.

(ii) we say that $\left(b^{n}\right)$ is a subseries basis if for each monotone increasing injection $\pi$, on the natural numbers, $\sum_{n=1}^{\infty} f^{\pi(n)}(x) b^{\pi(n)} \in E$ for all $x \in E$.

(iii) we say that $\left(b^{n}\right)$ is a bounded multiplier basis if for each $x \in E$ and each sequence, $\left(y_{n}\right)$ of scalars such that $\left|y_{n}\right| \leqq\left|f^{n}(x)\right|$ for all $n$, it follows that $\sum_{n=1}^{\infty} y_{n} b^{n} \in E$.

(1.2) Next we define six types of bases which, roughly speaking, fall into dual pairs corresponding to the obvious duality between $\left(b^{n}\right)$ and $\left(f^{n}\right)$.

(i) we say that $\left(b^{n}\right)$ is shrinking if $\left(f^{n}\right)$ is a Schauder basis for $E^{\prime}\left[\mathscr{T}_{b}(E)\right]$.

(i)' we say that $\left(b^{n}\right)$ is boundedly complete if $\sum_{n=1}^{\infty} x_{n} b^{n} \in E$ for every sequence of scalars, $\left(x_{n}\right)$ such that the sequence, $\left(\sum_{n=1}^{N} x_{n} b^{n}\right)_{N}$ is bounded in $E$.

(ii) we say that $\left(b^{n}\right)$ is of type $P$ if there is a neighborhood of $0, V$, in $E$ such that $b^{n} \in E-V$ for all $n$ and the sequence, $\left(\sum_{n=1}^{N} b^{n}\right)_{N}$ is bounded in $E$.

(ii) $)^{\prime}$ we say that $\left(b^{n}\right)$ is of type $P^{*}$ if the sequence $\left(b^{n}\right)_{n}$ is bounded in $E$ and the sequence, $\left(\sum_{n=1}^{N} f^{n}\right)_{N}$ is strongly bounded in $E^{\prime}$.

(iii) we say that $\left(b^{n}\right)$ is monotone if there is a fundamental system of neighborhoods of $0, \mathscr{V}$, in $E$ such that for each $V \in \mathscr{V}, x \in E, \rho_{V}\left(\sum_{n=1}^{N} f^{n}(x) b^{n}\right)$ is an increasing function of $N$, where $\rho_{V}$ is the gage of $V$.

(iii)' we say that $\left(b^{n}\right)$ is an $e$-Schauder basis if the sequence, $\left(S_{n}\right)$ in $\mathscr{L}(E, E)$, defined by $S_{N}(x)=\sum_{n=1}^{N} f^{n}(x) b^{n}$, is equicontinuous.

(1.3) The concepts of shrinking and boundedly complete bases were introduced by R. C. James [9] for Banach spaces although the terms seem to be due to Day [6]. Bases of type $P$ and type $P^{*}$ were introduced and extensively discussed in the case of Banach spaces by I. Singer [23]. See also [15]. 
(1.4) It is well known that for bases in a locally convex space, $E$, bounded multiplier implies subseries implies unconditional and if $E$ is sequentially complete, all three definitions are equivalent (see Day [6, p. 59]). In general however, we shall see below that it is easy to construct a wide class of examples for which none of the converse implications are valid.

2. The coordinate bases. In this section we observe that sequence spaces always have Schauder bases in one topology or another and relate the definitions of the previous section to various properties of sequence spaces. This will provide us with a useful technique for constructing large classes of examples and counterexamples.

(2.1) Let $\lambda, \mu$ be sequence spaces such that $\mu \subset \lambda^{\beta}$. Then $\langle\lambda, \mu\rangle$ is a dual system and it is obvious that $\left(e^{n}\right)$ is a Schauder basis for $\lambda\left[\mathscr{T}_{s}(\mu)\right]$. This of course will not be true for all dual system topologies, even when $\mu=\lambda^{\times}$(consider $l^{\infty}\left[\mathscr{T}_{b}\left(l^{1}\right)\right]$ ). We mention below one result in this direction, but clearly the general area is still open. First however, let us consider the definitions of (1.2), (1.3) in the simplest case.

(2.2) THEOREM. Let $\lambda$ be a sequence space and $E=\lambda\left[\mathscr{T}_{s}\left(\lambda^{\times}\right)\right]$. Then,

(i) $\left(e^{n}\right)$ is an unconditional basis for $E$.

(ii) $\left(e^{n}\right)$ is a subseries basis for $E$ if and only if $\lambda$ contains each of its stepspaces.

(iii) $\left(e^{n}\right)$ is a bounded multiplier basis for $E$ if and only if $\lambda$ is normal.

(iv) $\left(e^{n}\right)$ is shrinking if and only if for each $u \in \lambda^{\times}$, the sequence, $\left(u^{N}\right)$ of initial sections of $u$ is $\mathscr{T}_{b}\left(\lambda, \lambda^{\times}\right)$-convergent to $u$.

(v) $\left(e^{n}\right)$ is boundedly complete if and only if $\lambda$ is perfect.

(vi) $\left(e^{n}\right)$ is of type $P^{*}$ if and only if $\lambda^{\times}=l^{\infty}$.

(vii) $\left(e^{n}\right)$ is not of type $P$.

(viii) If $\left(e^{n}\right)$ is e-Schauder or monotone, then $\lambda^{\times}=\varphi$.

Proof. Statement (i) follows immediately from the fact that every absolutely convergent series of scalars is unconditionally convergent. Statement (ii) is an immediate consequence of the definitions involved. Statement (iii) is observed in [19]. We observe that the associated sequence of coefficient functionals corresponding to $\left(e^{n}\right)$ is again $\left(e^{n}\right)$ and that the sections of a sequence are precisely the partial sums of the representation of that sequence; with this remark, statement (iv) is easily seen to be a translation of the definition. Now suppose $\left(e^{n}\right)$ is boundedly complete and $x \in \lambda^{\times x}$. Then clearly, the sequence $\left(x^{N}\right)$ of sections of $x$ is a $\mathscr{T}_{s}\left(\lambda^{\times}, \lambda\right)$-bounded set, so by the above remark and the definition of boundedly complete, there is a $y \in \lambda$ such that $\left(x^{N}\right)$ is $\mathscr{T}_{s}\left(\lambda^{\times}, \lambda\right)$ convergent to $y$. That is, for each $u \in \lambda^{\times}, \lim \left\langle x^{N}, u\right\rangle=y$. In particular, for each $n, x_{n}=\lim _{N \rightarrow \infty}\left\langle x^{N}, e^{n}\right\rangle=\left\langle y, e^{n}\right\rangle$ $=y_{n}$, so $x=y \in \lambda$. Hence $\lambda$ is perfect. On the other hand, if $\lambda$ is perfect and $x$ is a sequence whose sections, $\left(x^{N}\right)$ form a $\mathscr{T}_{s}\left(\lambda, \lambda^{\times}\right)$-bounded set, then for each $u \in \lambda^{\times}$, there is a $v \in \lambda^{\times}$such that $x_{n} v_{n}=\left|x_{n} u_{n}\right|$ (since $\lambda^{\times}$is normal). But $\left(\sum_{n=1}^{N} x_{n} v_{n}\right)$ is by assumption bounded. Hence $\sum\left|x_{n} u_{n}\right|<\infty$ so we may conclude that $x \in \lambda^{\times \times}=\lambda$. Finally, it is obvious that $\left(x^{N}\right)$ is $\mathscr{T}_{s}\left(\lambda, \lambda^{\times}\right)$-convergent to $x$. This proves (v). Next 
we observe that if $u \in \lambda^{\times}$, then the sequence $\left(\left\langle e^{n}, u\right\rangle\right)$ is just $u$, so $\left(e^{n}\right)$ is bounded iff $\lambda^{\times} \subset l^{\infty}$. Now suppose $\lambda^{\times}=l^{\infty}$ and $B$ is a $\mathscr{T}_{s}\left(\lambda^{\times}, \lambda\right)$-bounded set. But $\lambda \subset \lambda^{\times \times}=l^{1}$, so $B$ is $\mathscr{T}_{s}\left(l^{\infty}, l^{1}\right)$-bounded and hence bounded in the $l^{1}$ norm, say,

$$
\sup \{\|b\|: b \in B\}<M \text {. }
$$

Then if $x \in B$,

$$
\left|\left\langle x, \sum_{n=1}^{N} e^{n}\right\rangle\right|=\left|\sum_{n=1}^{N} x_{n}\right| \leqq \sum_{n=1}^{N}\left|x_{n}\right| \leqq M \text { for all } N .
$$

Thus $\left(e^{n}\right)$ is of type $P^{*}$ for $\lambda$.

Conversely, if $\left(e^{n}\right)$ is of type $P^{*}$ we already know that $\lambda^{\times} \subset l^{\infty}$. Suppose $x \in \lambda$. Then if $u \in \lambda^{\times},\left(\sum_{n=1}^{N}\left|x_{n} u_{n}\right|\right)_{N}$ is bounded. This means that the sections of $|x|=\left(\left|x_{n}\right|\right)_{n}$ is a $\mathscr{T}_{s}\left(\lambda^{\times}, \lambda\right)$-bounded set. Therefore by the second condition defining type $P^{*}$ the sequence $\left(\sum_{n=1}^{N}\left|x_{n}\right|\right)_{N}=\left(\left\langle|x|^{N}, \sum_{n=1}^{N} f^{n}\right\rangle\right)_{N}$ is bounded so $x \in l^{1}$. Hence $\lambda \subset l^{1}$ so $l^{\infty} \subset \lambda^{\times}$and (vi) is proved. Next suppose that $\left(e^{n}\right)$ is of type $P$ for $E$. If $u \in \lambda^{\times}$, then since $\left(\sum_{n=1}^{N} e^{n}\right)$ is bounded and $\lambda^{\times}$is normal, $\sum_{n=1}^{\infty}\left|u_{n}\right|<\infty$ so $\lambda^{\times} \subset l^{1}$. On the other hand, the definition assures the existence of $u^{1}, \ldots, u^{k} \in \lambda^{\times}$such that for each $n$ there is an $i$ such that $\left|u_{n}^{i}\right|=\left|\left\langle b^{n}, u^{i}\right\rangle\right| \geqq 1$. Since $\lambda^{\times}$is normal and a vector space, it follows that there is a $u \in \lambda^{\times}$such that $\left|u_{i}\right| \geqq 1$ for all $i$. Hence $l^{\infty} \subset \lambda^{\times}$. Since $l^{1} \varsubsetneqq l^{\infty}$ we have a contradiction.

(2.3) The characterizations in the above theorem are useful in obtaining counterexamples and in pointing the way towards possible theorems. For example, we see easily that neither of the two implications of (1.4) are reversible. In the context of (2.2), we observe that if $\lambda$ is the vector space of all sequences having a finite range, then $\left(e^{n}\right)$ is a subseries basis but not a bounded multiplier basis, and if $\lambda$ is the vector space of all eventually constant sequences, then $\left(e^{n}\right)$ is unconditional but not subseries. The example, $\omega\left[\mathscr{T}_{s}(\varphi, \omega)\right]$ shows that for Fréchet spaces, a basis can be monotone and $e$-Schauder relative to the weak topology. That this cannot happen in Banach spaces is well known [18].

(2.4) Theorem (2.2) suggests a possible connection between bases and sequence spaces of considerably more generality. In the first place, one would like to interpret the types of bases for arbitrary topologies compatible with $\left\langle\lambda, \lambda^{\times}\right\rangle$and in the second place, one would like to know which locally convex spaces with bases are such that their duals can be identified with $\lambda^{\times}$. The next theorem and Theorem (2.7) give some results for the first question and the second is completely solved for bounded multiplier bases in the next section.

(2.5) ThEOREM. Let $\lambda$ be a sequence space and $E=\lambda[\mathscr{T}]$ with $E^{\prime}=\lambda^{\times}$. Then the statements (iii), (iv), (v), (vi) of (2.2) remain valid.

Proof. We have $\mathscr{T}_{s}\left(\lambda^{\times}, \lambda\right) \subset \mathscr{T} \subset \mathscr{T}_{k}\left(\lambda^{\times}, \lambda\right)$. We shall make use of a theorem of Köthe [10, p. 417], which says that if $\lambda$ is normal, $x \in \lambda$, then the sections, $\left(x^{N}\right)$ of $x$ converge to $x$ in the Mackey topology and hence in $\mathscr{T}$. Therefore if $\lambda$ is normal, 
$\mathscr{T}$-convergence of the expansion of $x$ relative to $\left(e^{n}\right)$ is equivalent to $x \in \lambda$. Hence it follows immediately that $\lambda$ is normal iff $\left(e^{n}\right)$ is a bounded multiplier basis for $E$. Statements (iv), (vi) follow from the fact that the definitions of shrinking and type $P^{*}$ involve only the strong topology and bounded sets. Since these are concepts which depend only on the dual system and not on which topology is involved (so long as it is compatible), we may apply (2.2).

Finally, if $\lambda$ is perfect, then $\left(e^{n}\right)$ is $\mathscr{T}_{s}\left(\lambda^{\times}, \lambda\right)$ boundedly complete. So, if the sections of $x$ are bounded, they are weakly bounded so $x \in \lambda$; thus the sections converge to $x$. If $\left(e^{n}\right)$ is boundedly complete, it is clearly $\mathscr{T}_{s}\left(\lambda^{\times}, \lambda\right)$-boundedly complete so $\lambda$ is perfect.

(2.6) The theorem of Köthe referred to in the above proof is very important in the theory of sequence spaces and its proof as given in [10] requires machinery such as the theory of projective limits and various characterizations of sequence spaces. We shall now show how a considerably more general result about sequence spaces is a trivial consequence of McArthur's generalization of the Orlicz-Pettis theorem [12]: If $E$ is locally convex, then a series has the property that every subseries converges, if and only if the series has the same property in the weak topology.

(2.7) THEOREM. If $\lambda$ is a sequence space, then $\lambda$ contains each of its stepspaces if and only if $\left(e^{n}\right)$ is a subseries basis for any topology compatible with $\left\langle\lambda, \lambda^{\times}\right\rangle$. In particular, sections are Mackey convergent.

Theorem 2.7 is merely a rephrasing of the Orlicz-Pettis-McArthur theorem above.

(2.8) The original version of (2.7) in [19] has been previously used by the authors to give an example of a weak Schauder basis which is not a Schauder basis. The proof given in [19] is entirely different from that above. That the theorem follows immediately from the OPM theorem was pointed out to the authors by Professor McArthur.

3. The sequence spaces determined by a basis.

(3.1) It was essentially observed by Banach [2] that if $E[\mathscr{T}]$ is a locally convex space and $\left(b^{n}\right)$ is a Schauder basis, one can define the following sequence spaces:

$$
\begin{aligned}
& \lambda=\lambda_{E}=\left\{x=\left(x_{n}\right) \mid \sum_{n=1}^{\infty} x_{n} b^{n} \in E[\mathscr{T}]\right\}, \\
& \mu=\mu_{E}=\left\{u=\left(u_{n}\right) \mid \sum_{n=1}^{\infty} u_{n} f^{n} \in E^{\prime}\left[\mathscr{T}_{s}(E)\right]\right\} .
\end{aligned}
$$

In this section we shall determine the relationship between $\mu$ and $\lambda^{\times}$. When there is no possibility of confusion, we shall identify $\lambda, \mu$ with $E, E^{\prime}$ respectively. However, for use in more delicate situations, we introduce the linear bijections, $\imath: \lambda \rightarrow E$, $\imath^{\prime}: E^{\prime} \rightarrow \mu$ defined in the obvious way from the relation, (1). Clearly $\varphi \subset \lambda \cap \mu$, $\langle\lambda, \mu\rangle$ is a dual system with the usual bilinear functional and $\imath, \imath^{\prime}$ are adjoints of each other and are weakly continuous [19]. 
(3.2) THEOREM. The space $\mu$ is always contained in $\lambda^{\beta}$.

Proof. If $u \in \mu, x \in \lambda$, then there exists $\bar{x} \in E, \bar{u} \in E^{\prime}$ such that $\bar{x}=\imath(x), u=r^{\prime}(\bar{u})$. Then $\langle\bar{x}, \bar{u}\rangle=\langle\imath(x), \bar{u}\rangle=\left\langle x, \imath^{\prime}(\bar{u})\right\rangle=\langle x, u\rangle$ which shows that $\sum x_{n} u_{n}$ converges so $u \in \lambda^{\beta}$.

(3.3) In order to apply the results of the previous section, the most pleasant situation would be if $\mu=\lambda^{x}$. Unfortunately, as we shall see below, (3.2) is, in general, the best possible result. We can, however, in the case of bounded multiplier bases, completely characterize the desired situation.

(3.4) THEOREM. Let $E[\mathscr{T}]$ be a locally convex space with a bounded multiplier basis, $\left(b^{n}\right)$. Then $\mu=\lambda^{\times}$if and only if $E^{\prime}$ is $\mathscr{T}_{s}\left(E, E^{\prime}\right)$-sequentially complete.

Proof. Suppose $\mu=\lambda^{\times}$. Then by (2.5) and the fact that $\left(b^{n}\right)$ is bounded multiplier, it follows that $\lambda$ is normal. Hence by a theorem of Köthe and Toeplitz [11], it follows that $\lambda^{\times}$is $\mathscr{T}_{s}\left(\lambda, \lambda^{\times}\right)$-complete. Therefore $E^{\prime}$ is $\mathscr{T}_{s}\left(E, E^{\prime}\right)$-sequentially complete. Conversely, if $\mu$ is $\mathscr{T}_{s}(\lambda, \mu)$-sequentially complete and $u \in \lambda^{\times}$, the sections of $u$ are $\mathscr{T}_{s}(\lambda, \mu)$-Cauchy and hence converge to $u$, so $u \in \mu$ and $\lambda^{\times} \subset \mu$. Since $\left(b^{n}\right)$ is a bounded multiplier basis, it follows that $\lambda^{\times}=\lambda^{\beta}$, so by (3.2), $\lambda^{\times} \subset \mu \subset \lambda^{\beta}=\lambda^{\times}$.

(3.5) In general, $\mu$ is strictly contained in $\lambda^{\beta}$ and $\mu, \lambda^{\times}$are not comparable. We give the corresponding examples.

(i) if $\lambda$ is a nonnormal sequence space (e.g., the set of convergent sequences) and $E[\mathscr{T}]=\lambda\left[\mathscr{T}_{s}\left(\lambda^{\times}\right)\right]$, then $\mu=\lambda^{\times} \varsubsetneqq \lambda^{\beta}$;

(ii) same as (i) but with $E[\mathscr{T}]=\lambda\left[\mathscr{T}_{s}\left(\lambda^{\beta}\right)\right]$. Then $\lambda^{\times} \varsubsetneqq \lambda^{\beta}=\mu$.

(iii) $E[\mathscr{T}]=l^{1}\left[\mathscr{T}_{s}\left(c_{0}\right)\right]$. Then $\mu=c_{0} \varsubsetneqq l^{\infty}=\lambda^{\times}$;

(iv) a product of the three preceding examples gives a single case in which all relations except (3.2) fail.

(3.6) We end this section with the observation that theorem (3.4) applies to any quasi-barrelled space with a bounded multiplier basis and to any Fréchet space with an unconditional basis (see Day [6, p. 59]). In particular, Theorems (2.5), (2.7) give nontopological characterizations of several bases types for these cases.

\section{Dual PaIRS OF TYPES OF BASES}

\section{Shrinking and boundedly complete bases.}

(1.1) I. Singer [23] has observed the following duality relationship between shrinking and boundedly complete bases. Let $\left(b^{n}\right)$ be a basis for a Banach space, $E=E[\mathscr{T}]$. Then

A. $\left(b^{n}\right)$ is shrinking if and only if $\left(f^{n}\right)$ is a boundedly complete basis for $E^{\prime}\left[\mathscr{T}_{b}(E)\right]$.

B. $\left(b^{n}\right)$ is boundedly complete if and only if $\left(f^{n}\right)$ is a shrinking basis for $H=\left[f^{n}\right]$ where $H$ is the $\mathscr{T}_{b}(E)$-closed subspace of $E^{\prime}$ generated by $\left(f^{n}\right)$ equipped with the topology induced by $E^{\prime}\left[\mathscr{T}_{b}(E)\right]$.

In the general case only one of the four implications embodied in A, B remains 
true. In this section we prove the one, give counterexamples for the other three and then mention several situations in which they are true, thereby generalizing A, B to a considerable extent. Finally we show how the methods introduced in this paper yield a generalization of an important theorem of James.

(1.2) ExAmples. (i) Let $E[\mathscr{T}]=l^{1}\left[\mathscr{T}_{k}\left(c_{0}\right)\right]$. Clearly $\lambda=l^{1}, \mu=c_{0}$ and $\left(e^{n}\right)$ is a shrinking basis for $E$ since $\left(e^{n}\right)$ is a basis for the normed space, $c_{0}$, which is $E^{\prime}\left[\mathscr{T}_{b}(E)\right]$. However $\mu^{\times}=\lambda$ and $c_{0}$ is not perfect, so by $\mathrm{I}(2.5),\left(e^{n}\right)$ is not boundedly complete for $c_{0}$. Hence, one implication in $\mathrm{A}$ is, in general, false.

(ii) Consider the dual system $\langle\varphi, \varphi\rangle$. Let $E=\varphi\left[\mathscr{T}_{s}(\varphi)\right]$. Then $E^{\prime}\left[\mathscr{T}_{b}(E)\right]=\varphi\left[\mathscr{T}_{b}(\varphi)\right]$ which is a dense subspace of $\omega\left[\mathscr{T}_{b}(\varphi, \omega)\right]$. Hence, $\left(E^{\prime}\left[\mathscr{T}_{b}(E)\right]\right)^{\prime}=\varphi=E$. Now all this means that if we take (as usual), $b^{n}=f^{n}=e^{n}$, then $H$, as defined in B above is $E^{\prime}$ and $\left(e^{n}\right)$ is a Schauder basis for $\left(E^{\prime}\left[\mathscr{T}_{b}(E)\right]\right)^{\prime}$. Hence, $\left(f^{n}\right)$ is shrinking for $H$. However, $\left(\sum_{n=1}^{N} e^{n}\right)_{N}$ is clearly bounded in $E$ but does not converge, so $\left(b^{n}\right)$ is not a boundedly complete basis.

On the other hand, if $E=l^{\infty}\left[\mathscr{T}_{k}\left(l^{1}, l^{\infty}\right)\right], b^{n}=e^{n}=f^{n}$, then since $\lambda=l^{\infty}, \mu=l^{1}=\lambda^{\times}$ and $\lambda$ is perfect, it follows from $\mathrm{I}(2.5)$ that $\left(b^{n}\right)$ is boundedly complete. Also, $E^{\prime}=l^{1}$, so it follows from $\mathrm{I}(2.5)$ again that $\left(f^{n}\right)$ is not shrinking for $H=l^{1}$ since sections in $l^{\infty}=\mu^{\times}$are not strongly convergent. Hence, both implications in B are, in general, false.

(1.3) Let us recall that if $\left(f^{n}\right)$ is a Schauder basis for $E^{\prime}\left[\mathscr{T}_{b}(E)\right]$, then $\left(b^{n}\right)$ is shrinking.

(1.4) THEOREM. Let E[T] be a locally convex space with a Schauder basis $\left(b^{n}\right)$ and suppose that every $\mathscr{T}_{b}\left(E, E^{\prime}\right)$-bounded sequence is equicontinuous. Then if $\left(b^{n}\right)$ is shrinking, $\left(f^{n}\right)$ is a boundedly complete basis for $E\left[\mathscr{T}_{b}(E)\right]$.

Proof. Suppose $\left(a_{n}\right)$ is a sequence of scalars such that $\left(\sum_{n=1}^{N} a_{n} f^{n}\right)_{N}$ is $\mathscr{T}_{b}\left(E, E^{\prime}\right)$ bounded. We define

$$
S=\left\{\sum_{n=k}^{k+p} a_{n} f^{n} \mid k, p=1,2, \ldots\right\}
$$

Then clearly $S$ is $\mathscr{T}_{b}\left(E, E^{\prime}\right)$-bounded and hence equicontinuous. Hence, if $x=\sum_{n=1}^{\infty} x_{n} b^{n} \in E$, and $\varepsilon>0$, there exists $N$ such that

$$
\left|\left\langle\sum_{n=N}^{\infty} x_{n} b^{n}, \quad \sum_{n=k}^{k+p} a_{n} f^{n}\right\rangle\right| \leqq \varepsilon \text { for all } k, p .
$$

In particular, for any $p>0$,

$$
\left|\left\langle x, \quad \sum_{n=N}^{N+p} a_{n} f^{n}\right\rangle\right|=\left|\left\langle\sum_{n=N}^{\infty} x_{n} b^{n}, \quad \sum_{n=N}^{N+p} a_{n} f^{n}\right\rangle\right| \leqq \varepsilon
$$

which shows that $\left(\sum_{n=1}^{N} a_{n} f^{n}\right)_{N}$ is a $\mathscr{T}_{s}\left(E, E^{\prime}\right)$-Cauchy sequence in an equicontinuous set, so it converges weakly to $f \in E^{\prime}$ by the Alaoglu-Bourbaki Theorem. Since $\left(b^{n}\right)$ is shrinking, there is a sequence, $\left(a_{n}^{\prime}\right)$ is of scalars such that $f=\sum_{n=1}^{\infty} a_{n}^{\prime} f^{n}$ in 
$E^{\prime}\left[\mathscr{T}_{b}(E)\right]$ and hence in $E^{\prime}\left[\mathscr{T}_{s}(E)\right]$. Since each $b_{n}$ is $\mathscr{T}_{s}\left(E, E^{\prime}\right)$-continuous it follows that $a_{n}=a_{n}^{\prime}$ for all $n$ and we are finished.

(1.5) We remark that by combining (1.3) and (1.4) one obtains the equivalence A for a wide class of spaces which includes not only the quasi-barrelled spaces, but also the $\mathscr{D} \mathscr{F}$-spaces [8]. Next we consider the equivalence, B.

(1.6) THEOREM. Let $E[\mathscr{T}]$ be a barrelled space with a Schauder basis $\left(b^{n}\right)$. If $\left(b^{n}\right)$ is boundedly complete, then $\left(f^{n}\right)$ is shrinking for $H$ (as defined in II(1.1)).

Proof. Consider the map $\Phi: E \rightarrow H^{\prime}$ defined by $\Phi(x)(u)=\langle x, u\rangle, x \in E, u \in H$. Since $\Phi\left(b^{n}\right)\left(f^{n}\right)=1$ and $\left(b^{n}\right)$ is a basis, it follows that $\Phi$ is $1-1$. Now let $v \in E^{\prime}$. If $x \in E$, then $x=\sum_{n=1}^{\infty} f^{n}(x) b^{n}$, so the sequence of scalars, $\left(\sum_{n=1}^{N} f^{n}(x)\left\langle b^{n}, v\right\rangle\right)_{N}$ is bounded. Hence, the sequence $\left(\sum_{n=1}^{N}\left\langle b^{n}, v\right\rangle f^{n}\right)_{N}$ is $\mathscr{T}_{s}\left(E, E^{\prime}\right)$-bounded and hence $\mathscr{T}_{b}\left(E, E^{\prime}\right)$-bounded since $E$ is barrelled. Thus it is a bounded sequence of the locally convex space, $H$. Therefore, if $y \in H^{\prime}$, we have $\left(\sum_{n=1}^{N}\left\langle b^{n}, v\right\rangle\left\langle y, f^{n}\right\rangle\right)_{N}$ is a bounded sequence of scalars. Thus we have shown that the sequence

$$
\left(\sum_{n=1}^{N}\left\langle y, f^{n}\right\rangle b^{n}\right)_{N}
$$

is $\mathscr{T}_{s}\left(E^{\prime}, E\right)$-bounded and hence bounded in $E[\mathscr{T}]$. Since $\left(b^{n}\right)$ is boundedly complete there exists $x \in E$ such that $x=\sum_{n=1}^{\infty}\left\langle y, f^{n}\right\rangle b^{n}$. Clearly, $\Phi(x)=y$ so we have shown that $\Phi$ is an algebraic isomorphism.

To show that $\left(f^{n}\right)$ is shrinking for $H$, it remains only to show that $\Phi\left(b^{n}\right)$ is a basis for $H^{\prime}\left[\mathscr{T}_{b}(H)\right]$. For this, it is sufficient to show that $\mathscr{T}_{b}(H)$ is weaker than the topology, $\Phi[\mathscr{T}]$. But this is clear since $\mathscr{T}=\mathscr{T}_{b}\left(E^{\prime}\right)$ and every $\mathscr{T}_{s}\left(H^{\prime}, H\right)$-bounded set is also $\mathscr{T}_{s}\left(E, E^{\prime}\right)$-bounded.

(1.7) It is clear from the proof of the last result that the condition, $E[\mathscr{T}]$ is barrelled, can be replaced by the slightly weaker condition: every $\mathscr{T}_{s}(E, H)$ bounded set is $\mathscr{T}$-equicontinuous.

(1.8) TheOREM. Let E[T] be a locally convex space with a Schauder basis $\left(b^{n}\right)$ and suppose $E$ is $\mathscr{T}_{s}(H, E)$-sequentially complete. Then if $\left(f^{n}\right)$ is a shrinking basis for $H,\left(b^{n}\right)$ is boundedly complete.

Proof. Let $S=\left(\sum_{n=1}^{N} a_{n} b^{n}\right)_{N}$ be bounded in $E[\mathscr{T}]$, and let $u \in H$; then $u=\sum_{n=1}^{\infty}\left\langle b^{n}, u\right\rangle f^{n}$ in $H$, so if $\varepsilon>0$, there is $N_{0}$ such that $N \geqq N_{0}$ implies that for each $M \geqq N$,

$$
\begin{aligned}
\left|\left\langle\sum_{n=N}^{M} a_{n} b^{n}, u\right\rangle\right| & =\left|\sum_{n=1}^{M} a_{n}\left\langle b^{n}, u\right\rangle-\sum_{n=1}^{N} a_{n}\left\langle b^{n}, u\right\rangle\right| \\
& =\left|\left\langle\sum_{n=1}^{M} a_{n} b^{n}, u-\sum_{n=1}^{N}\left\langle b^{n}, u\right\rangle f^{n}\right\rangle\right| \leqq \varepsilon .
\end{aligned}
$$

Hence $S$ is a $\mathscr{T}_{s}(H, E)$-Cauchy sequence so there exists $x \in E$ such that $x=\lim _{N} \sum_{n=1}^{N} a_{n} b^{n}$. Hence $\left(b^{n}\right)$ is boundedly complete. 
(1.9) By combining (1.7) and (1.8) we may conclude that the equivalence B remains valid for any locally convex space, $E[\mathscr{T}]$ such that every $\mathscr{T}_{s}(E, H)$-bounded set is $\mathscr{T}$-equicontinuous and $E$ is $\mathscr{T}_{s}(H, E)$ sequentially complete.

We close this section with a generalization of an important theorem of $\mathbf{R}$. C. James [9] for Banach spaces. The proof is a major example of how the results of I can be used to apply the theory of sequence spaces in a positive way to the theory of Schauder bases in locally convex spaces.

(1.10) THEOREM. Let E[T] be a locally convex space with a bounded multiplier basis $\left(b^{n}\right)$ and suppose that $E^{\prime}$ is $\mathscr{T}_{s}\left(E, E^{\prime}\right)$-sequentially complete. Then $\left(b^{n}\right)$ is boundedly complete if and only if $E$ is $\mathscr{T}_{s}\left(E^{\prime}, E\right)$-sequentially complete.

Proof. In view of I(3.4), with $\lambda=\lambda_{E}$ as defined in I(3.1), we may apply I(2.5), (v) and translate the conclusion of the theorem to read: a sequence space, $\lambda$, is perfect if and only if it is $\mathscr{T}_{s}\left(\lambda^{\times}\right)$-sequentially complete.

This is a well-known theorem (Köthe [10, p. 415]).

(1.11) The previous result is not true if $\left(b^{n}\right)$ is not a bounded multiplier basis. In fact an example of James [9] gives a space which is not weakly sequentially complete, but has a boundedly complete basis.

\section{Bases of type $P$ and type $P *$.}

(2.1) I. Singer [23] has observed the following duality relationship between bases of types $P$ and $P^{*}$. Let $\left(b^{n}\right)$ be a basis for a Banach space, $E=E[\mathscr{T}]$. Then

C. $\left(b^{n}\right)$ is of type $P$ if and only if $\left(f^{n}\right)$ is a basis of type $P^{*}$ for $H$.

D. $\left(b^{n}\right)$ is of type $P^{*}$ if and only if $\left(f^{n}\right)$ is a basis of type $P$ for $H$.

E. A Banach space has a basis of type $P$ if and only if it has a basis of type $P^{*}$.

Singer has also observed the following results for these types of bases [9].

F. If $E[\mathscr{T}]$ is a Banach space, then $E[\mathscr{T}]$ has an unconditional basis of type $P$ if and only if $E[\mathscr{T}]$ is isomorphic to $c_{0}$ (usual norm).

G. A Banach space, $E[\mathscr{T}]$ has an unconditional basis of type $P^{*}$ if and only if $E[\mathscr{T}]$ is isomorphic to $l^{1}$ (usual norm).

We have only partial information regarding the duality theorems $\mathrm{C}, \mathrm{D}, \mathrm{E}$. We mention only $c_{0}\left[\mathscr{T}_{s}\left(l^{1}, c_{0}\right)\right]$ for which $\left(e^{n}\right)$ is not a basis of type $P$ (I(2.2), (vii)), but $\left(e^{n}\right)$ is a basis of type $P^{*}$ for $l^{1}\left[\mathscr{T}_{b}\left(c_{0}\right)\right]=l^{1}\left[\mathscr{T}_{k}\left(l^{\infty}\right)\right](\mathrm{I}(2.5))$. Also, $\mathrm{I}(2.2)$ (vi) and (vii) show that $E$ is not, in general, valid. Conditions for the other implications embodied in C, D, E to hold remain open questions, although it is easy to see that our methods yield some results in special cases.

We are able to give a variant of $\mathrm{G}$ and a proof of $\mathrm{F}$ which is completely different from that employed by Singer [23].

(2.2) Proof of F. One way is clear; for the other, let $\left(b^{n}\right)$ be an unconditional Schauder basis for $E[\mathscr{T}]$ which is of type $P^{*}$. Since $E[\mathscr{T}]$ is a Banach space, we have by $\mathrm{I}(3.3), E[\mathscr{T}]=\lambda[\mathscr{T}],(\lambda[\mathscr{T}])^{\prime}=\lambda^{\times}$. Hence, by $\mathrm{I}(2.5), \lambda^{\times}=l^{\infty}$ and $\lambda$ is $\mathscr{T}_{s}\left(l^{\infty}\right)$-dense in $l^{1}$, hence $\mathscr{T}_{k}\left(l^{\infty}\right)=\mathscr{T}_{b}\left(l^{\infty}\right)$-dense in $l^{1}$. That is, $\lambda=l^{1}$. 
(2.3) THEOREM. Let $E[\mathscr{T}]$ be a locally convex space with a Schauder basis, $\left(b^{n}\right)$ of type $P$ such that $\mu=\lambda^{\times}$. Then $E$ is not $\mathscr{T}_{s}\left(E^{\prime}\right)$-sequentially complete.

Proof. If $E$ were $\mathscr{T}_{s}\left(E^{\prime}\right)$-sequentially complete, then [10, p. 415] it would follow that $\lambda$ would be perfect so by $\mathrm{I}(2.5),\left(b^{n}\right)$ would be a boundedly complete basis. Then, if $\left(b^{n}\right)$ were of type $P$, it would follow that $\sum_{n=1}^{\infty} b^{n}$ were convergent, so $\lim _{n} b^{n}=0$ which contradicts the other part of the definition of type $P$.

(2.4) Lemma. If $E[\mathscr{T}]$ is a Banach space with an unconditional basis then $E$ is $\mathscr{T}_{s}\left(E^{\prime}\right)$-sequentially complete if and only if $E[\mathscr{T}]$ has no subspace isomorphic to $c_{0}$.

An elegant proof of (2.4) is given in [3].

(2.5) To see the relationship between (2.3) and F, observe that (2.3), (2.4) together yield the fact that a Banach space with an unconditional basis of type $P$ has a subspace isomorphic to $c_{0}$.

\section{Monotone and $e$-Schauder bases.}

(3.1) Russo [21] has observed that a Schauder basis is $e$-Schauder if and only if it is monotone.

We shall have need of the following result [18]:

(3.2) Lemma. Let $E[\mathscr{T}]$ be a locally convex space with a Schauder basis, $\left(b^{n}\right)$, such that $\left(b^{n}\right)$ is e-Schauder. Then $A \subset E$ is precompact if and only if $\lim _{N} \sum_{n=1}^{N} f^{n}(x) b^{n}$ $=x$, uniformly for all $x \in A$.

(3.3) THEOREM. If $\left(b^{n}\right)$ is a boundedly complete e-Schauder basis for E[T्T] then $E[\mathscr{T}]$ is sequentially complete.

Proof. Let $\left(x_{k}\right)$ be a Cauchy sequence in $E$ and let $a_{n}=\lim _{k}\left\langle x_{k}, f^{n}\right\rangle$ which clearly exists for each $n$. We shall first show that $\left(\sum_{n=1}^{N} a_{n} b^{n}\right)_{N}$ is bounded in $E$.

Since $\left(x_{k}\right)$ is Cauchy, it is precompact so by (3.2), the set, $\left(\sum_{n=1}^{N} f^{n}\left(x_{k}\right) b^{n}\right)_{N, k}$ is bounded in $E$. Let $u \in E^{\prime}$ and $M>0$ such that for all $N, k,\left|\left\langle\sum_{n=1}^{N} f^{n}\left(x_{k}\right) b^{n}, u\right\rangle\right| \leqq M$. Now fix $N$ and choose $k_{0}$ such that $\left|a_{n}-\left\langle x_{k_{0}} f^{n}\right\rangle\right| \cdot\left|\left\langle b^{n}, u\right\rangle\right| \leqq 1 / N, n=1, \ldots, N$. Then

$$
\begin{aligned}
\left|\left\langle\sum_{n=1}^{N} a_{n} b^{n}, u\right\rangle\right| \leqq & \left|\left\langle\sum_{n=1}^{N}\left(a_{n}-\left\langle x_{k_{0}}, f^{n}\right\rangle\right) b_{n}, u\right\rangle\right| \\
& +\left|\sum_{n=1}^{N}\left\langle f^{n}\left(x_{k_{0}}\right) b^{n}, u\right\rangle\right| \\
& \leqq \sum_{n=1}^{N}\left|a_{n}-\left\langle x_{k_{0}}, f^{n}\right\rangle\right| \cdot\left|\left\langle b^{n}, u\right\rangle\right|+M \\
& \leqq M+1 .
\end{aligned}
$$

Hence $\left(\sum_{n=1}^{N} a_{n} b^{n}\right)_{N}$ is bounded so there exists $x \in E$ such that $x=\sum_{n=1}^{N} a_{n} b^{n}$. We 
shall show that $x=\lim _{k} x_{k}$. Let $V$ and $W$ be absolutely convex neighborhoods of 0 in $E$ with $W+W+W \subset V$. Repeating the above argument, we can obtain $N_{1}$ such that for each $k$,

$$
x_{k}-\sum_{n=1}^{N_{1}} f^{n}\left(x_{k}\right) b^{n} \in W, x-\sum_{n=1}^{N_{1}} a_{n} b^{n} \in W .
$$

Then choose $k_{0}$ such that $k \geqq k_{0}$ implies

$$
\sum_{n=1}^{N_{1}}\left(f^{n}\left(x_{k}\right)-a_{n}\right) b^{n} \in W .
$$

Adding these three inequalities yields $x-x_{k} \in V$ for $k \geqq k_{0}$, and the theorem is proved.

(3.4) Without assuming that $\left(b^{n}\right)$ is an $e$-Schauder basis one cannot obtain the result. In fact if $J$ is the space of R. C. James [9] mentioned in (1.11), $J^{*}$ has a boundedly complete basis but is not weakly sequentially complete.

\section{Reflexivity.}

\section{Applications}

(1.1) One of the main reasons for introducing shrinking and boundedly complete bases was to study reflexivity. These concepts proved very useful in the case of Banach spaces (see [9]). Of course for more general locally convex spaces, one must also study semireflexivity. The first results along this line are due to Retherford [16] and are quoted below (1.2). In this section we obtain complete characterizations of reflexivity and semireflexivity under reasonably general conditions which include Fréchet spaces with unconditional bases.

(1.2) THEOREM. (i) If $E[\mathscr{T}]$ is a reflexive, complete locally convex space with a Schauder basis, $\left(b^{n}\right)$, then the basis is both shrinking and boundedly complete.

(ii) If $E[\mathscr{T}]$ is a locally convex space with a Schauder basis that is both shrinking and boundedly complete, then $E[\mathscr{T}]$ is semireflexive.

(1.3) LEMMA. Let $E[\mathscr{T}]$ be a locally convex space with a boundedly complete basis, $\left(b^{n}\right)$ and suppose $\mu=\lambda^{\times}$(see $\left.\mathrm{I}(3.1)\right)$. Then $\lambda$ is perfect and $\left(b^{n}\right)$ is a bounded multiplier basis.

Proof. The proof is immediate from $\mathrm{I}(2.5)$ and the fact that every perfect sequence space is normal.

(1.4) THEOREM. Let E[T] be a locally convex space with a Schauder basis, $\left(b^{n}\right)$ and suppose $\mu=\lambda^{\times}$. Then

(i) $E[\mathscr{T}]$ is semireflexive if and only if $\left(b^{n}\right)$ is both shrinking and boundedly complete.

(ii) $E[\mathscr{T}]$ is reflexive if and only if $\left(b^{n}\right)$ is both shrinking and boundedly complete, $\left(b^{n}\right)$ is a basis for $E\left[\mathscr{T}_{b}\left(E^{\prime}\right)\right]$, and $\mathscr{T}=\mathscr{T}_{k}\left(E^{\prime}\right)$.

Proof. (i) One implication is immediate from (1.2), (ii). If, on the other hand, 
$E[\mathscr{T}]$ is semireflexive, let $B=\left(\sum_{n=1}^{N} a_{n} b^{n}\right)_{N}$ be bounded in $E[\mathscr{T}]$. Then $B$ is $\mathscr{T}_{s}\left(E^{\prime}\right)$ relatively compact. Hence, there exists $x=\sum_{n=1}^{\infty} f^{n}(x) b^{n}$ in $E[\mathscr{T}]$ which is a weak limit point of $B$. Since each $f^{n}$ is weakly continuous, it follows that $f^{n}(x)$ is a limit point of $f^{n}(B)=\left\{a_{n}\right\}$. That is, $f^{n}(x)=a_{n}$. Hence, $\left(b^{n}\right)$ is boundedly complete. By (1.3), it follows that $\lambda$ is perfect so we may apply [10, §30, 7.(1), 7.(4)] to conclude that sections in $\lambda^{\times}$are $\mathscr{T}_{b}\left(\lambda, \lambda^{\times}\right)$-convergent. Hence by $(2.5),\left(b^{n}\right)$ is shrinking.

(ii) To say that $\left(b^{n}\right)$ is a basis for $E\left[\mathscr{T}_{b}\left(E^{\prime}\right)\right]$ is to say that sections in $\lambda$ are strongly convergent. Hence if $E[\mathscr{T}]$ is reflexive, we may conclude as above that $\lambda$ is perfect and apply [10, $\$ 30,7 .(1), 7 .(5)]$, to conclude that $\left(b^{n}\right)$ is a basis for $E\left[\mathscr{T}_{b}\left(E^{\prime}\right)\right]$. The other conclusions follow from the fact that reflexivity implies that $E$ is both semireflexive and barrelled.

On the other hand, we can conclude that $E[\mathscr{T}]$ is semireflexive from (i) and by $[10, \S 30,7 .(1)]$ that $E\left[\mathscr{T}_{k}\left(E^{\prime}\right)\right]$ is barrelled. Hence $E[\mathscr{T}]=E\left[\mathscr{T}_{k}\left(E^{\prime}\right)\right]$ is reflexive.

(1.5) We remark that the conditions of the previous theorem are always satisfied by a Fréchet (and hence Banach) space with an unconditional basis. This is because we always have $\mu=\lambda^{\times}$(see I(3.6)).

The next theorem shows that for conditional boundedly complete bases (1.4) is never applicable.

(1.6) THEOREM. Let E[T $[$ be a locally convex space with a conditional boundedly complete basis, $\left(b^{n}\right)$. Then $\mu \neq \lambda^{\times}$and $\left(e^{n}\right)$ is not a boundedly complete basis for $\lambda\left[\mathscr{T}_{s}\left(\lambda^{\times}\right)\right]$.

Proof. If $\mu=\lambda^{\times}$, then by $\mathrm{I}(2.5), \lambda$ is perfect and hence normal so again by $\mathrm{I}(2.5),\left(b^{n}\right)$ is a bounded multiplier basis; hence $(\mathrm{I}(1.4))$ an unconditional basis for $E[\mathscr{T}]$ which is a contradiction.

If $\left(e^{n}\right)$ is boundedly complete for $\lambda\left[\mathscr{T}_{s}\left(\lambda^{\times}\right)\right]$, then $\lambda$ is perfect and hence normal so $\lambda^{\beta}=\lambda^{\times}$. Hence by I(3.2), $\mu \subset \lambda^{\times}$. Also, if $x=\sum x_{n} b^{n} \in E[\mathscr{T}]$, and $\left|y_{n}\right| \leqq\left|x_{n}\right|$ for all $n$, then $\sum_{n=1}^{N} y_{n} b^{n}$ is $\mathscr{T}_{s}\left(\lambda^{\times}, \lambda\right)$-convergent, so it is $\mathscr{T}_{s}(\mu, \lambda)$-convergent. Thus there exists $y=\sum a_{n} b^{n} \in E[\mathscr{T}]$ such that $\sum_{n=1}^{N} y_{n} b^{n}$ is $\mathscr{T}_{s}\left(E^{\prime}, E\right)$-convergent to $y$. By the usual argument, $a_{n}=y_{n}$ and we have shown that $\left(b^{n}\right)$ is a bounded multiplier and hence unconditional basis for $E[\mathscr{T}]$ which is a contradiction.

The hypotheses of (1.6) are realized in $J^{*}$, where $J$ is again the space of James.

\section{Similar bases.}

(2.1) In studying locally convex spaces with bases, a natural area of investigation is the situation in which one has a locally convex space with two Schauder bases. In particular, one would consider the case in which the corresponding sequence spaces are different. This turns out to be a very deep question related to infinite matrices, summability theory, etc. and will be treated in a subsequent paper. There is a dual problem of some interest which can be studied by our methods. Suppose we consider two different locally convex topologies on a vector space with a subset that is a basis for both.

Specifically, if $E, F$ are locally convex spaces with Schauder bases, $\left(b^{n}\right),\left(c^{n}\right)$ 
respectively, we say, using the notation of $\mathrm{I}(3.1)$ that the bases are similar (cosimilar) if $\lambda_{E}=\lambda_{F}\left(\mu_{E}=\mu_{F}\right)$. We may interpret this situation by saying that we have a sequence space, $\lambda(\mu)$ and topologies $\mathscr{T}_{1}, \mathscr{T}_{2}$ such that $\left(e^{n}\right)$ is a Schauder basis for $\lambda\left[\mathscr{T}_{1}\right]$ and $\lambda\left[\mathscr{T}_{2}\right]\left(\left(e^{n}\right)\right.$ is a $\mathscr{T}_{s}\left(\lambda_{i}, \mu\right)$-Schauder basis, $\left.i=1,2\right)$.

Interesting results regarding these concepts have been obtained by Arsove and Edwards [1], Retherford and Jones [20] and Davis [5].

The first question we ask is whether the types of bases defined in I(1.1), (1.2) are affected by replacing a basis with a similar basis. It is easy to verify that each of the types defined in $\mathrm{I}(1.1)$ are independent of the choice of a similar basis. However in the case of the six types defined in $\mathrm{I}(1.2)$ the situation is much more interesting. In general, the answer is that all six types may be satisfied by one basis and not by a similar basis. However if $\mu=\lambda^{\times}$we occasionally get a positive answer. The detailed results follow.

(2.2) THEOREM. Let $E\left[\mathscr{T}_{1}\right], F\left[\mathscr{T}_{2}\right]$ be locally convex spaces with Schauder bases $\left(b^{n}\right),\left(c^{n}\right)$ which are similar and suppose $\mu_{E}=\mu_{F}=\lambda_{E}^{\times}$. Then $\left(b^{n}\right)$ is shrinking (resp. boundedly complete, resp. $\left.P^{*}\right)$ if and only if $\left(c^{n}\right)$ is also.

Proof. This is immediate from the characterizations in $\mathrm{I}(2.5)$ since none of them mention the topology but refer only to the dual system $\left\langle\lambda, \lambda^{\gamma}\right\rangle$.

(2.3) We now list the counterexamples for the general case. In view of II(3.1), we may ignore monotone bases. In all cases, $b^{n}=c^{n}=e^{n}$.

(i) Shrinking. $E\left[\mathscr{T}_{1}\right]=l^{1}\left[\mathscr{T}_{s}\left(c_{0}\right)\right], F\left[\mathscr{T}_{2}\right]=l^{1}\left[\mathscr{T}_{s}\left(l^{\infty}\right)\right]$. Clearly, $\left(e^{n}\right)$ is a basis for $c_{0}\left[\mathscr{T}_{b}\left(l^{1}\right)\right]$ but not for $l^{\infty}\left[\mathscr{T}_{b}\left(l^{1}\right)\right]$.

(ii) Boundedly complete. $E\left[\mathscr{T}_{1}\right]=\varphi\left[\mathscr{T}_{s}(\omega)\right], F\left[\mathscr{T}_{2}\right]=\varphi\left[\mathscr{T}_{s}(\varphi)\right] .\left(e^{n}\right)$ is boundedly complete for $E\left[\mathscr{T}_{1}\right]$ by $\mathrm{I}(2.5)$. But the sequence $\left(\sum_{n=1}^{N} e^{n}\right)_{N}$ is bounded in $F\left[\mathscr{T}_{2}\right]$ and does not converge.

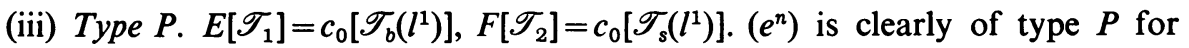
$E\left[\mathscr{T}_{1}\right]$ but by $\mathrm{I}(2.2)$, not for $F\left[\mathscr{T}_{2}\right]$.

(iv) Type $P^{*} . E\left[\mathscr{T}_{1}\right]=l^{1}\left[\mathscr{T}_{b}\left(l^{\infty}\right)\right], F\left[\mathscr{T}_{2}\right]=l^{1}\left[\mathscr{T}_{s}(\varphi)\right] .\left(e^{n}\right)$ is of type $P^{*}$ for $E\left[\mathscr{T}_{1}\right]$ by $\mathrm{I}(2.5)$, but not for $F\left[\mathscr{T}_{2}\right]$ since $\left(\sum_{n=1}^{N} e^{n}\right)_{N}$ is not $\mathscr{T}_{b}\left(l^{1}, \varphi\right)$-bounded. In fact, $\left(\sum_{n=1}^{M} e^{n}\right)_{M}$ is $\mathscr{T}_{s}\left(\varphi, l^{1}\right)$-bounded, but $\left(\left\langle\sum_{n=1}^{M} e^{n}, \sum_{n=1}^{N} e^{n}\right\rangle\right)_{N, M}$ is an unbounded set of numbers.

(v) e-Schauder. $E\left[\mathscr{T}_{1}\right]=l^{1}\left[\mathscr{T}_{b}\left(l^{\infty}\right)\right], F\left[\mathscr{T}_{2}\right]=l^{1}\left[\mathscr{T}_{s}\left(l^{\infty}\right)\right] .\left(e^{n}\right)$ is clearly $e$-Schauder for $E\left[\mathscr{T}_{1}\right]$ but not for $F\left[\mathscr{T}_{2}\right]$ by $\mathrm{I}(2.2)$.

(2.4) Observe that in the previous number, both (iii), (v) satisfy the hypotheses in (2.2) so that the theorem cannot be extended to the cases of type $P$ and $e$-Schauder. The results of the previous section suggest that sharper results might be obtained by considering both similarity and co-similarity. Before doing this, we observe that by considering $l^{1}\left[\mathscr{T}_{s}\left(l^{\infty}\right)\right]$ and $l^{1}\left[\mathscr{T}_{s}\left(c_{0}\right)\right]$ we obtain similar bases which are not co-similar and from $l^{\infty}\left[\mathscr{T}_{s}\left(l^{1}\right)\right], c_{0}\left[\mathscr{T}_{s}\left(l^{1}\right)\right]$ we obtain co-similar bases which are not similar.

We can however obtain some positive results in this direction. 
(2.5) THEOREM. Let E, $F$ be locally convex spaces with bounded multiplier similar bases, $\left(b^{n}\right),\left(c^{n}\right)$ respectively. Suppose further that $E^{\prime}, F^{\prime}$ are weakly sequentially complete. Then $\left(b^{n}\right),\left(c^{n}\right)$ are co-similar.

Proof. In view of I(3.4) and our assumptions, we have $\mu_{E}=\lambda_{E}^{\times}=\lambda_{F}^{\times}=\mu_{F}$.

(2.6) COROLlary. In the context of (2.5) it follows that $E$ and $F$ are weakly (and hence Mackey) isomorphic.

Proof. From (2.5) it follows that $b^{(n)}, c^{(n)}$ are both similar and co-similar. This clearly implies the conclusion.

(2.7) As a final application we give an easy proof of a theorem of H. S. Collins originally proved by completely different methods.

(2.8) THeOREM. A locally convex space $E[\mathscr{T}]$ is isomorphic to $l^{\infty}\left[\mathscr{T}_{k}\left(l^{1}\right)\right]$ if and only if the following conditions are satisfied:

(i) E[T] has a Schauder basis, $\left(b^{n}\right)$, which is similar to $\left(e^{n}\right)$ as a Schauder basis for $l^{\infty}\left[\mathscr{T}_{k}\left(l^{1}\right)\right]$.

(ii) $\left(f^{n}\right)$ is $\mathscr{T}_{b}\left(E, E^{\prime}\right)$-bounded.

(iii) Every sequentially continuous linear form on $E$ is continuous.

(iv) $\mathscr{T}=\mathscr{T}_{k}\left(E^{\prime}\right)$.

Proof. Suppose $E[\mathscr{T}]$ satisfies (i), (ii), and (iii). Then by (2.1), $\left(b^{n}\right)$ is a bounded multiplier basis. Hence, $\mu_{E} \subset\left(\lambda_{E}\right)^{\beta}=\left(\lambda_{E}\right)^{\times}=\left(l^{\infty}\right)^{\times}=l^{1}$. We must show that $l^{1} \subset \mu_{E}$. In fact, if $u \in l^{1}$, we can define $g: E \rightarrow K$ by $g(x)=\sum_{n=1}^{\infty} u_{n} f^{(n)}(x)$. Clearly $g$ is linear. Let $\left(x^{k}\right)$ be a sequence converging to 0 in $E[\mathscr{T}]$. Hence, by (ii), there exists $M>0$ such that $\left|f^{n}\left(x^{k}\right)\right| \leqq M$ for all $n, k$. Let $\varepsilon>0$. Choose $b$ such that

$$
\sum_{n=b+1}^{\infty}\left|u_{n}\right| \leqq \frac{\varepsilon}{M}
$$

Then

$$
\left|g\left(x^{k}\right)\right| \leqq\left|\sum_{n=1}^{b} u_{n} f^{n}\left(x^{k}\right)\right|+\left(\sum_{n=b+1}^{\infty}\left|u_{n}\right|\right) M \leqq\left|\sum_{n=1}^{b} u_{n} f^{n}\left(x^{k}\right)\right|+\varepsilon .
$$

Since $\left(x^{k}\right)$ is a null sequence and $f^{1}, \ldots, f^{b} \in E^{\prime}$, it follows that there exists $k_{0}$ such that $k \geqq k_{0}$ implies, $\left|g\left(x^{k}\right)\right| \leqq 2 \varepsilon$. Hence, by (iii), $g \in E^{\prime}$. That is, $u \in \mu_{E}$. Hence $\mu_{E}=l^{1}$, so $\left(b^{n}\right)$ is similar and co-similar to $\left(e^{n}\right)$, so $E$ is Mackey isomorphic with $l^{\infty}$ and the result follows from (iv).

On the other hand, it is obvious that $l^{\infty}\left[\mathscr{T}_{k}\left(l^{1}\right)\right]$ satisfies (i), (ii) and (iv). To see that (iii) is satisfied first observe that in $l^{1}, \mathscr{T}_{s}\left(l^{\infty}\right)$ and $\mathscr{T}_{k}\left(l^{\infty}\right)$ convergent sequences coincide. Hence by a result of Köthe $[10, \S 30,7 .(6)], \mathscr{T}_{s}\left(l^{1}\right)$ and $\mathscr{T}_{k}\left(l^{1}\right)$ convergent sequences in $l^{\infty}$ also coincide. Now $l^{\infty}\left[\mathscr{T}_{k}\left(l^{1}\right)\right]$ is complete $[10, \S 30,5 .(9)]$ and separable, so by [22, p. 150, Corollary 3] condition (iii) holds. 


\section{BIBLIOGRAPHY}

1. M. Arsove and R. Edwards, Generalized bases in topological linear spaces, Studia Math. 19 (1960), 95-113.

2. S. Banach, Théorie des operations lineaires, Monogr. Mat., Warsaw, 1932.

3. C. Bessaga and A. Pełczynski, On subspaces of a space with an absolute basis, Bull. Acad. Polon. Sci. 6 (1958), 313-315.

4. N. Bourbaki, Éléments de mathematique, Livre V, Espaces vectoriels topologiques, Chapitre IV, Actualités Sci. Ind. No. 1139, Hermann, Paris, 1955.

5. W. Davis, Dual generalized bases in linear topological spaces, Proc. Amer. Math. Soc. 17 (1966), 1057-1063.

6. M. M. Day, Normed linear spaces, Springer-Verlag, Berlin, 1962.

7. J. Dieudonné, On biorthogonal systems, Michigan Math. J. 2 (1953/1954), 7-20.

8. A. Grothendieck, Sur les espaces $(F)$ et (DF), Summa Brasil. Math. 3 (1954), 57-123.

9. R. C. James, Bases and reflexivity of Banach spaces, Ann. of Math. 52 (1950), 518-527.

10. G. Köthe, Topologische lineare Raume. I, Springer-Verlag, Berlin, 1960.

11. G. Köthe and O. Toeplitz, Lineare Raume mit unendlich vielen Koordinaten und Ringe undendlicher Matrizen, J. Reine Angew. Math. 171 (1934), 193-226.

12. C. W. McArthur, On a theorem of Orlicz and Pettis, Pacific J. Math. (to appear).

13. - A convergence criterion with applications to series in locally convex spaces, Duke Math. J. 34 (1967), 193-200.

14. A Pelczynski and M. Kadec, Basic sequences, biorthogonal systems and norming sets in Banach and Fréchet spaces, Studia Math. 25 (1965), 297-323. (Russian)

15. V. Ptak, Biorthogonal systems and reflexivity of Banach spaces, Czechoslovak. Math. J. 9 (1959), 319-326.

16. J. R. Retherford, Bases, basic sequences and reflexivity of linear topological spaces, Math. Ann. 248 (1966), 280-285.

17. J. R. Retherford and C. W. McArthur, Some remarks on bases in linear topological spaces, Math. Ann. 247 (1966), 38-41.

18. — Uniform and equicontinuous Schauder bases of subspaces, Canad. J. Math. 17 (1965), 207-212.

19. J. R. Retherford and Ed Dubinsky, Schauder bases in compatible topologies, Studia Math. 28 (1967), 221-226.

20. J. R. Retherford and O. Jones, Similar bases in barrelled spaces, Proc. Amer. Math. Soc. 18 (1967), 677-680.

21. J. P. Russo, Monotone and e-Schauder bases of subspaces, Dissertation, Florida State Univ., Tallahasse, 1965.

22. H. H. Schaefer, Topological vector spaces, Macmillan, New York, 1966.

23. Ivan Singer, Basic sequences and reflexivity of Banach spaces, Studia Math. 21 (1962), 351-369.

24. —_ On the basis problem in linear topological spaces, Rev. Math. Pures Appl. 10 (1965), 453-457.

\footnotetext{
Tulane University of Louisiana, New Orleans, Louisiana

The Louisiana State University, Baton ROUGE, LOUISIANA
} 